\title{
5G Networks: Challenges and Techniques for Energy Efficiency
}

\author{
Madeha Azhar \\ Electronics Department \\ NED University of Engineering and Technology \\ Karachi, Pakistan \\ madiha_azhar@hotmail.com
}

\author{
Amna Shabbir \\ Electronics Department \\ NED University of Engineering and Technology \\ Karachi, Pakistan \\ amna06@gmail.com
}

\begin{abstract}
The growing importance of energy efficient networks with high data rate requirements is a major concern for network operators. Services provided by the network operators are required to ensure the consumers' satisfaction. For the providing of high data rates with good signal quality, small cells are deployed. But these cells can increase energy consumption if not equipped with some intelligent power saving or distribution mechanism. In this paper, a previously tested small cell sleeping mode scheme is compared with the new proposed scheme of reducing power in low or normal traffic hours. This scheme provided $13-15 \%$ increase in energy efficiency. The new scheme resulted to beneficial simulated outcomes and can be applied to overcome the energy consumption issue.
\end{abstract}

Keywords-green communication; energy efficiency; power distribution

\section{INTRODUCTION}

There is a huge growth in the usage of cell phones and the number of their users. Devices like cell phones and tablets have become very handy and are available to almost everyone. There has been a tremendous growth in the number of cell phone subscribers requiring high data rates as well as speedy internet for faster browsing. The social media era brought the need of higher data rates. Mobile operators, while trying to fulfill the data traffic and to accommodate every subscriber with acceptable quality of service, left the energy consumption issue at backend. Service providers came up with the solution to deploy Base Stations (BSs) in large quantity to provide higher data rates to the subscribers. Network energy consumption rapidly increases when large numbers of BSs are deployed to provide the demanded services to every user. As the number of BSs increased a large amount of power was required for the huge macro BSs to operate which resulted in increased energy consumption and increased $\mathrm{CO}_{2}$ emission. Heterogeneous networks were deployed having different cell sizes like macro, micro, pico and femto cells which have limited coverage areas providing services in allocated areas with substantial data rates and signal quality [1].

Recently, the concern went towards energy saving. In recent years many of the researchers provided expectable solutions and techniques helpful to achieve energy efficiency
[2-5]. Energy efficiency can be achieved with several ways: by enhancing the performance of different hardware components, by turning off selected BS components, by the deployment of efficient heterogeneous networks, br using natural energy resources. By turning off selectively BS components, some energy can be conserved. Power amplifiers on the other hand, are the main source of energy consumption in a BS, so, by improving the power amplifier performance, power consumption can be decreased. Other techniques can also be implemented in order to achieve energy efficient networks but the quality of the provided service must not be jeopardized.

\section{RELATED WORK}

\section{A. Network Basics}

Mobile telephone systems have been through a major upgrade in the last few years and this development has resulted not only in the increase of mobile subscribers but also of their demands According to the trend of mobile telephone systems, after the subscriber connection request, the device is connected to the BS with the strongest signal at the given time. To understand and implement any technique involving mobile networks there are several important parameters that must be clearly understood. Turning towards the heterogeneous networks, the simulation for each tier becomes difficult as the network complexity increases with the number of subscribers [6]. For example the macro BSs are systematically distributed and have large coverage areas with higher transmitting powers in the range of $40 \mathrm{~W}$ with large antenna gains. On the contrary, there are femto cells that are deployed with extreme efficiency and smaller coverage zones, evenly the femto cells can be placed in homes and small offices with central planning, with power in the range of $250 \mathrm{~mW}$. Between these two extremes pico cells are deployed, mainly in the peak or huge traffic areas with limited coverage areas and, as estimated, 10 picos per macro cell is a suitable configuration. The pico cells are not randomly placed neither in a regular pattern and their transmit power is in the range of $250 \mathrm{~mW}-2 \mathrm{~W}$. There are two ways to obtain energy efficiency in a cellular network. One way is to reduce the power consumption of the main energy consumers that are the power amplifiers and other hardware components by improving the performance of different hardware 
components or by using advanced software. The second method is the intelligent placement of BSs in addition with the low power nodes.

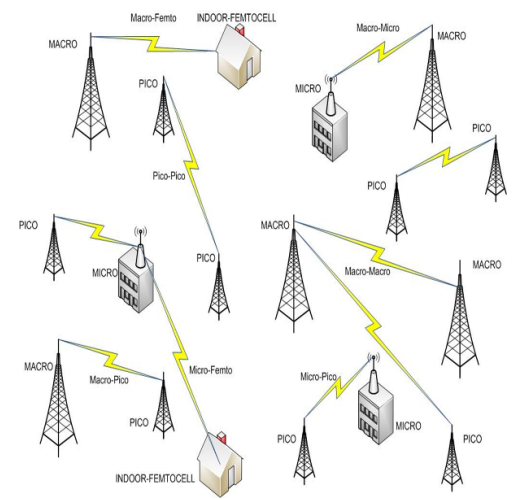

Fig. 1. Heterogeneous cellular network topology incorporating different forms of small cell deployments as an overlay on the macrocell network.

\section{B. Deployment of Small Cells}

The small cells can operate in two major modes: open access mode, in which every user trying to connect is allowed access with a security check while in the closed access mode the access is given only to the registered user or to the subscriber authenticated by a procedure. There is another operating mode, the hybrid access mode, in which very limited resources are available to the users. Substantial energy savings can be achieved by controlling the access mechanism and the sleep mode procedures. During the sleep mode of a BS, the user must be verified by the small cell circuitry or by a specific procedure which decides whether to provide service or not. Choosing to place an unutilized BS into sleep mode is an efficient and direct way to help the energy saving in a network, but to implement sleep mode, one must first ensure the good signal quality of the other subscribers that are handed over to other cells. Sleep mode in a cell brings coverage problems that must be considered $[2,5,7]$. There are two types of techniques of using the sleep mode in order to decrease the energy consumption of a network.

- $\quad$ Sleep mode implementation in macro BSs

- Switching off small BSs when not in use

For adopting small cell BS sleeping modes different techniques and algorithms can be implemented:

- Small cell controlled sleep mode

- Core network control sleep mode

- User equipment (UE) control sleep mode

There has been a tradeoff in discharging traffic to macrocell and energy utilization of small cell. For implementing sleep mode procedures in macro BSs some of the studies showed that the energy consumption of a network can be significantly reduced while retaining the proper coverage and user power utilization criteria. Macro BSs are considered to provide extensive coverage and hence their power consumption is huge. Adopting sleep mode in femto cell BS can help in maintaining coverage but cannot contribute in energy saving. Applying sleep mode in the macro BSs can significantly reduce the energy consumption but can affect the coverage adversely. So this is tradeoff [7] between maintaining the coverage and the energy consumption by applying sleep mode in macro and femto BSs (FBSs). Authors in [6] proposed an efficient solution of power conservation for huge number of femto cells in sleeping span. It is proposed that if femto cell cluster deployment has extensive coverage then it results in lesser energy usage which allows different femto BSs to adopt various sleeping modes while macroBSs serve to activate the FBSs.

\section{NETWORK MODEL}

The considered network is a long term evolution network cellular model that is a three tier heterogeneous network. It comprises of the macrocells with the smaller size in surroundings and pico cells deployed inside houses for limited coverage to the indoor users hence indoor users are provided with femtocell access points. In highly populated areas giant macrocells are placed which are enough to provide coverage to a large number of users and the small cells are present in the surroundings to provide a BS for the traffic that is offloaded from macro BSs and to increase network capacity to maximize the throughput. In the rest of the paper, preference is given to the power consumption performance of the smaller cell deployments and the femto cells are deployed inside homes for restricted use. The macro and pico cells are considered for evaluating the network performance. The numbers of macrocell and picocell sites are denoted by $\mathrm{N}_{\text {macro }}$ and $\mathrm{N}_{\text {pico }}$ respectively. It is considered in the given scenario that there are 70,000 mobile subscribers present in the area under evaluation with a total of 20,000 users uniformly distributed outdoors and 50,000 indoors. The indoor user distribution is calculated on an average of 5 users located in every household, so that there will be a maximum of 10,000 picocells to be deployed in all households. The subscribers located outside and inside are allowed to have the same traffic requirements that are between $500 \mathrm{Mbps}$ and $900 \mathrm{Mbps}$.

- Assumption 1. There is no interference within the cell in the downlink.

- Assumption 2. For FBSs the closed access mode is selected, i.e. FBSs behave as regular BSs and are in reach to the registered user equipment (UE) only. This access mode is considered to be used widely for households and small coverage area.

- Assumption 3. Each UE can be served by at most one BS. Cell selection is based on a maximum downlink received power of pilot signal.

A single picocell in the network is considered to draw $P_{\text {pico }}$ $=15 \mathrm{~W}$ from the main power socket, can transmit up to $0.25 \mathrm{~W}$ and can serve five simultaneous users. Each macrocell site is comprised of three sectors that require a total power of $\mathrm{P}_{\text {macro }}$ $=3 \mathrm{~kW}$. Dynamic power saving modes are not considered here. For macrocells an average spectral efficiency of $1.7 \mathrm{~b} / \mathrm{s} / \mathrm{Hz}$ per macrocell sector is considered [7] and a $20 \mathrm{MHz}$ carrier bandwidth. 
The total energy consumption per annum (=8760 hours) of the network can be calculated by (1):

$$
\mathrm{E}_{\text {network }}=\left(\mathrm{N}_{\text {macro }} \cdot \mathrm{P}_{\text {macro }}+\mathrm{N}_{\text {pico }} \cdot \mathrm{P}_{\text {pico }}\right) \cdot 8760
$$

$\mathrm{E}_{\text {network }}$ is calculated in $\mathrm{Wh}$ and it is assumed that the core network components can be neglected for both macro and pico cells as they contribute very less to the total energy consumption. The combined voice and data traffic modeling of each subscriber is considered to be have a duty cycle of $17.15 \%$.

TABLE I. NETWORK PARAMETERS

\begin{tabular}{|c|c|c|}
\hline Serial Number & Symbols & Description \\
\hline 1. & $\mathrm{~N}_{\text {macro }}$ & $\begin{array}{l}\text { Number of macrocell } \\
\text { sites }\end{array}$ \\
\hline 2. & $\mathrm{~N}_{\text {pico }}$ & Number of picocell sites \\
\hline 3. & $\mathrm{P}_{\text {pico }}$ & $\begin{array}{c}\text { Power of a picocell or } \\
\text { small cell }\end{array}$ \\
\hline 4. & $\mathrm{P}_{\text {macro }}$ & Power of macrocell \\
\hline 5. & $E_{\text {network }}$ & $\begin{array}{l}\text { Average network energy } \\
\text { consumption per annum }\end{array}$ \\
\hline 6. & $\mathrm{E}_{\mathrm{e}}$ & Energy efficiency \\
\hline 7. & $\mathrm{R}$ & $\begin{array}{l}\text { Average date rate } \\
\text { provided of a base } \\
\text { station }\end{array}$ \\
\hline 8. & $\mathrm{P}_{\mathrm{C}}$ & Consumed power \\
\hline 9. & $\mathrm{P}_{\mathrm{S}}$ & Signal power \\
\hline 10. & $\mathrm{P}_{\mathrm{N}}$ & Noise power \\
\hline 11. & Coverage $_{\text {smallcell }}$ & $\begin{array}{c}\text { Small cell coverage } \\
\text { detection by hardware }\end{array}$ \\
\hline 12. & $\mathrm{~S}_{\mathrm{N}}$ & $\begin{array}{l}\text { Sniffer detection of rise } \\
\text { in uplink power }\end{array}$ \\
\hline 13. & RPS & Received power strength \\
\hline 14. & $\mathrm{~T}_{\mathrm{H}}$ & Threshold power \\
\hline 15. & SC_power & $\begin{array}{l}\text { Small cell initial } \\
\text { processing and pilot } \\
\text { signal transmission }\end{array}$ \\
\hline 16. & UE_ID & $\begin{array}{l}\text { To check user is } \\
\text { registered or not }\end{array}$ \\
\hline 17. & TIN & Traffic intensity \\
\hline
\end{tabular}

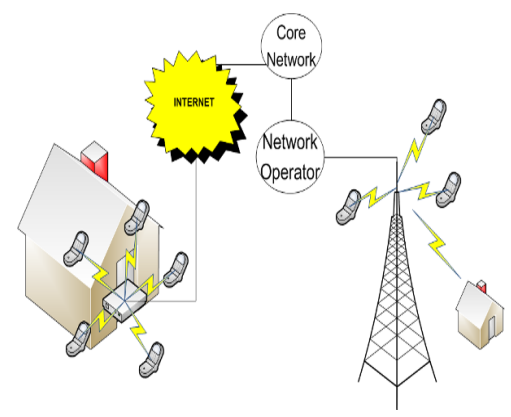

Fig. 2. Macrocell and femtocell connections

There are three sleep mode schemes: small cell controlled sleep mode, core network controlled sleep mode and user equipment controlled sleep mode [8]. While examining these schemes it is observed that they are proposed only with the idea to shut down some of the specific hardware components that are not essential to remain constantly on, while the traffic pattern of the subscribers in the observed areas is not considered. All the areas have different user density and traffic pattern to determine the voice and data bits usage and demand.
In this paper, the traffic of the area under observation is considered diversive. The user traffic intensity is broken up in two parts that are the normal and peak traffic hours.

The macrocells are deployed in every area having high transmitting powers that can be utilized when UE could not find any small cell to provide coverage. Thus small cells have a low power sniffer capability that detects any active call from UE unit to the macrocell. When there is no active calls made by any UE, the small cell is inactive and incase of any active users in the coverage area the small cells are activated. As the UE unit is detected inside the sensing range of small cell sniffer, the received power is increased. As the UE transmits at high power to connect to the macrocell so this rise in power is easily detectable and when UE is very close to the small cell, its pilot transmission is activated. If the requested user is allowed to the small cell so a macro to small cell handover takes place and the small cell serves the user, otherwise if the user is not allowed by the small cell, the macrocell serves the user. This process is repeated: for every user the small cell hardware determines the small cell traffic condition. The density of users is checked out periodically and when there is regular traffic load in a small cell, the power of cell is reduced to $40 \%$ of the total power delivered from the socket. When there is peak traffic load the power is $60 \%$ of the total power. If there are more users than the serving capability of small cell then some of the users are switched to be served by the nearby macrocell.

\section{A. Working Algorithm}

Definitions:

- Coverage $_{\text {smallcell }}=1$ means user is in small cell coverage

- $\quad \mathrm{SN}=1$ means sniffer detects rise in uplink power

- $\quad \mathrm{SC} \_$power=1 means small cell is activated and its pilot transmission is activated

- UE_ID $=1$ means the requested user is registered

- $\quad \mathrm{TIN}=1$ means traffic intensity is at normal condition and $\mathrm{TIN}=0$ means there is peak traffic

Algorithm:

Update Coverage smallcell $_{\text {and }} \mathrm{S}_{\mathrm{N}}$

if $\mathrm{RPS}>\mathrm{T}_{\mathrm{H}}$

update $\mathrm{SC}$ power $=1$

update $\mathrm{SC}$ _power to macrocell

if UE ID $=1$

assign $\mathrm{SC}$ _power $=1$

update TIN

$$
\begin{aligned}
& \text { if TIN }=1 \\
& \text { else } \mathrm{P}^{\prime}=0.40 * \mathrm{P}_{\text {pico }} \\
& \text { end } \quad \mathrm{P}^{\prime}=0.60 * \mathrm{P}_{\text {pico }}
\end{aligned}
$$

end

end 


\section{RESULTS}

\section{A. Users}

The total number of users indoors and outdoors and the maximum number of users are considered to be 70,000. Users require different amounts of data rates at different times comprising a varied pattern of traffic. Some times the data rate demand increases resulting in peak traffic hours. When the demand for data rate is under a threshold it is considered normal traffic hours. The number of users is plotted against the number of deployed pico cells in Figure 3. The scheme named user equipment and the small cell controlled sleep mode for the reduction of average network energy consumption are taken as a reference [8]. The proposed scheme suggests reducing the input power or the distributed power according to the traffic pattern. If the traffic pattern is normal then the power is reduced to $40 \%$ of the input power and if the users increase their demands the supplied becomes $60 \%$ of the input power.

\section{B. Energy}

A new parameter is calculated in this paper, the energy efficiency of the network. The energy efficiency parameter is very popular in determining the performance of the network and the most commonly used metric for the energy efficiency of a communication link is bit per joule, $\mathrm{E}_{\mathrm{e}}$ which is defined as the ratio of the total network throughput over the energy consumption within a given period, the unit of which is bits/Joule. Average network energy consumption is plotted in Figure 4 against the number of pico cells. Proposed normal and peak traffic hours power distribution scheme is applied to achieve energy saving. The normal traffic hour scheme provides the most efficient result but even in peak traffic the energy consumption is lower than that of sleep mode scheme. The graph of energy efficiency of pico cells considering different data rates, which is usually required these days is shown in Figure 5. The energy efficiency of the network with different number of cells declines as the pico cell density increases but at higher data rates the energy efficiency shows improvement. The energy efficiency is calculated using (2):

$$
E_{e}=\frac{R}{P_{c}}
$$

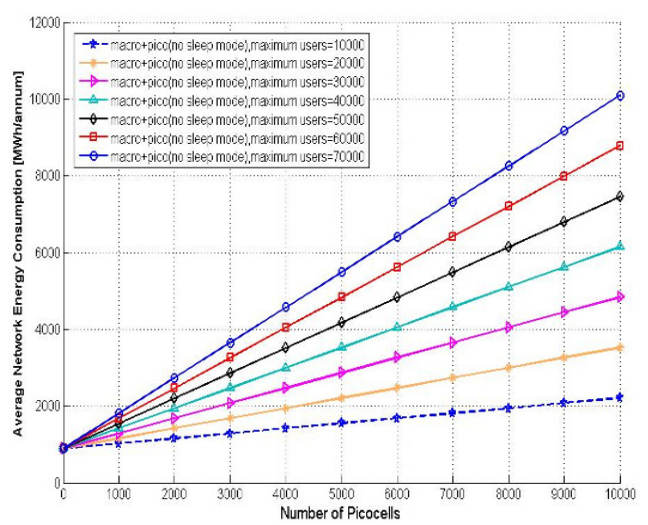

Fig. 3. Energy consumption with picocells for different numbers of users

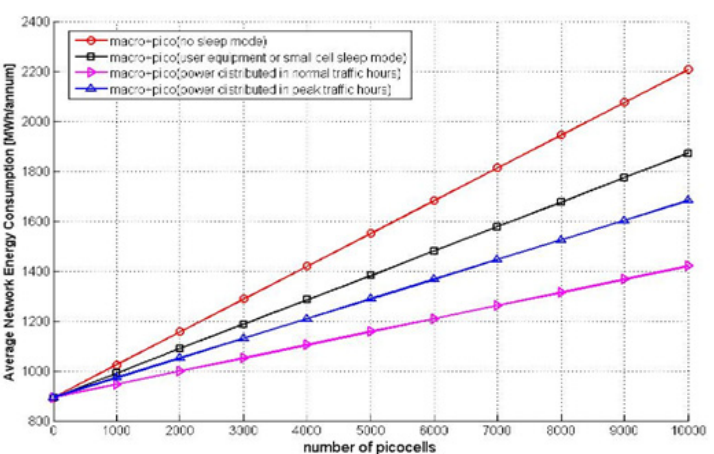

Fig. 4. Energy consumed with number of picocells for different schemes

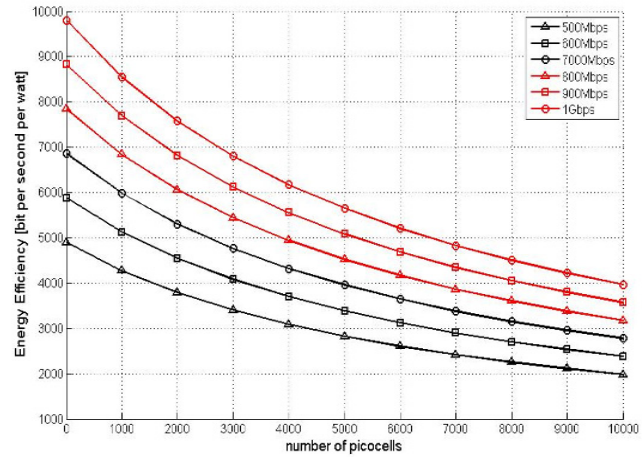

Fig. 5. Energy efficiency with number of picocells for different data rates

Energy efficiency is evaluated for four different schemes (no sleep mode, UE, normal and peak hour mode) using data rate of $900 \mathrm{Mbps}$ in Figure 6. The change in the consumed power in every scheme leads to the result that energy efficiency during normal traffic hours is higher and even in peak traffic hours the energy efficiency is still higher than that of the small cell controlled sleep mode.

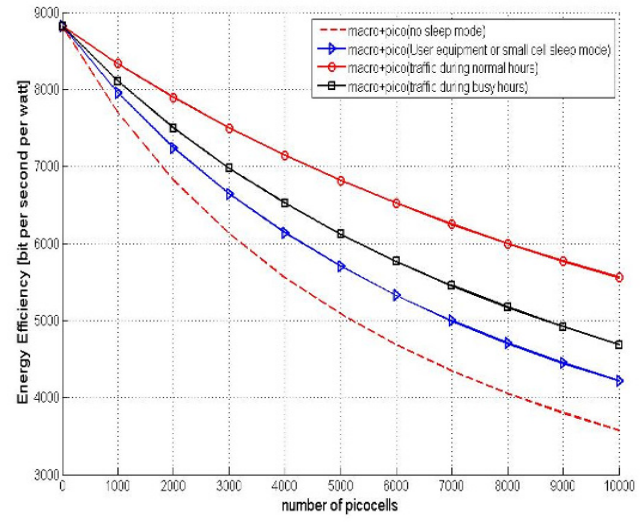

Fig. 6. Energy efficiency with number of picocells for different schemes at $900 \mathrm{Mbps}$

\section{Signal to Noise Ratio}

The signal to noise ratio is considered as one of the most important parameters of the communication system which is 
used to measure the effectiveness of the considered communication system or network. Moreover, the signal quality is also dependent on the value of SNR. SNR is the main factor of how the signal is received Big SNR means radio receiver sensitivity performance.

$$
S N R=\frac{\text { signal power }}{\text { noise power }} \text { or } S N R=\frac{P_{S}}{P_{N}}
$$

The energy efficiency is an important factor that needs to be monitored while the network is operational as much as when in rest mode. The energy efficiency against SNR is plotted in Figure 7 in decibels using different schemes. We see that, normal traffic hour scheme has the highest energy efficiency and the scheme with peak traffic even has energy efficiency greater than that of small cell controlled sleep mode.

\section{Comparison}

From Figure 6 we observe that that there is $14.7 \%$ improvement in the energy efficiency evaluation when small cell controlled scheme is compared with energy efficiency graph calculated during normal traffic, moreover there is $6.2 \%$ improvement when small cell controlled scheme is compared with energy efficiency graph calculated during peak traffic.

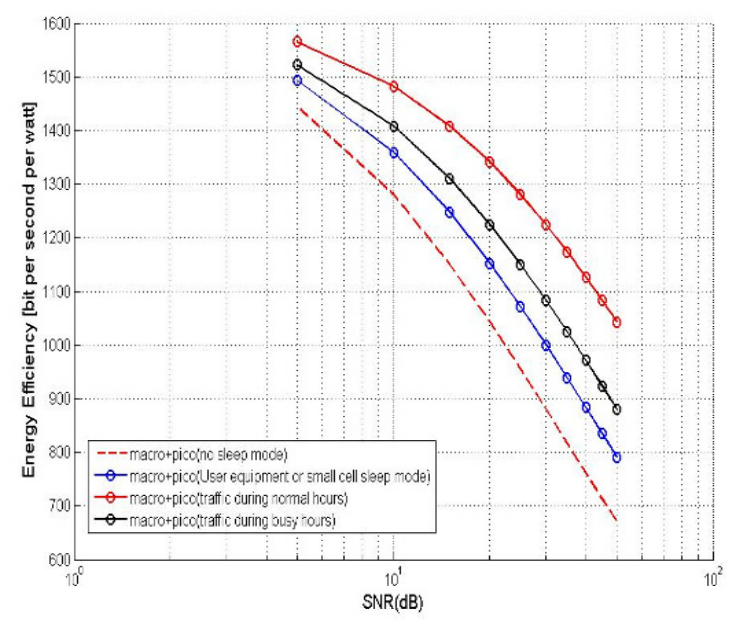

Fig. 7. Energy efficiency with SNR fot different schemes

\section{CONCLUSION}

The results discussed in this paper illustrate that there is considerable improvement in the energy efficiency for both scenarios of the proposed scheme, the normal traffic mode and the peak traffic mode. When the number of users increases there is an increase in the demand of data rate, so the macro and pico cells are operated at full loads. The proposed scheme provides finer and better results when plotted for the normal traffic mode in the reduction of the average energy consumption of all the base stations. Hence, it is concluded that the new proposed scheme of considering traffic pattern to reduce power supply according to the traffic mode gives profitable simulated results that show improvements in energy efficiency.

\section{REFERENCES}

[1] Y. S. Soh, T. Q. S. Quek, M. Kountouris, H. Shin, "Energy efficient heterogeneous cellular networks", IEEE Journal on Selected Areas in Communications, Vol. 31, No. 5, pp. 840-850, 2013

[2] J. Wu, Y. Zhang, M. Zukerman, E. K. N. Yung, "Energy-efficient base-stations sleep-mode techniques in green cellular networks: a survey", IEEE Communications Surveys \& Tutorials, Vol. 17, No. 2, pp. 803-826, 2015

[3] Y. Wang, Y. Zhang, Y. Chen, R. Wei, "Energy-efficient design of two-tier femtocell networks", EURASIP Journal on Wireless Communications and Networking, Vol. 2015, 2015

[4] M. Wildemeersch, T. Q. Quek, A. Rabbachin, C. H. Slump, A. Huang, "Energy efficient design of cognitive small cells", IEEE International Conference on Communications, pp. 2701-2706, Budapest, Hungary, June9-13, 2013

[5] J. Peng, P. Hong, K. Xue, "Stochastic analysis of optimal base station energy saving in cellular networks with sleep mode", IEEE Communications Letters, Vol. 18, No. 4, pp. 612-615, 2014

[6] F. Han, S. Zhao, S., L. Zhang, J. Wu, "Survey of strategies for switching off base stations in heterogeneous networks for greener 5G systems", IEEE Access, Vol. 4, pp. 4959-4973, 2016

[7] G. He, S. Zhang, Y. Chen, S. Xu, "Energy efficiency and deployment efficiency tradeoff for heterogeneous wireless networks", IEEE Global Communications Conference, Anaheim, CA, USA, pp. 3189-3194, December, 3-7, 2012

[8] I. Ashraf, F. Boccardi, L. Ho, "Power savings in small cell deployments via sleep mode techniques", IEEE 21st International Symposium on Personal, Indoor and Mobile Radio Communications Workshops, Instanbul, Turkey, pp. 307-311, September 26-30, 2010 